\title{
Finite-size effects on linear stability of pure-fluid convection
}

\author{
Yih-Yuh Chen* \\ Condensed Matter Physics 114-36, California Institute of Technology, Pasadena, California 91125
}

(Received 17 June 1991)

\begin{abstract}
The linear stability of pure-fluid Rayleigh-Bénard convection in a finite cell of arbitrary geometry can be formulated as a self-adjoint eigenvalue problem. This, when coupled with perturbation theory, allows one to deduce how the sidewalls affect its stability. In particular, it is shown that for almost all boundary conditions the difference between the onset Rayleigh number and its infinite-cell limit scales like $L^{-2}$ as the cell dimension $L$ tends to $\infty$, and near the sidewall the temperature and velocity are of order $L^{-1}$ compared to their bulk values. The validity of replacing the true thermal boundary condition by a frequently used mathematically simpler homogeneous one is also demonstrated.
\end{abstract}

PACS number(s): 47.25.Qv, 44.25.+f, 47.20.Bp

In recent years Rayleigh-Bénard convection has attracted many researchers because it has provided us with a system that is experimentally well controllable and has rich dynamics and pattern formation that are not only interesting in themselves but also seem to be shared by other systems such as chemical reaction-diffusion systems. Although progress in theoretical description of this system has been steady, relatively few analytical results concerning the effects of the sidewalls have been obtained. Because the influence of a confining wall can be very complex for a convection cell with complications from impurity or rotation, I have decided to investigate the boundary effects for the simplest system-pure-fluid convection-as a first step towards an analytical understanding of this problem. It should be pointed out that the results derived here are quite general in the sense that the geometry of the convection cell is not restricted, and the boundary conditions considered are realistic.

The study of the convective instability of pure fluid is easier because the problem has a variational character. A variational formulation of the linear stability of pure-fluid Rayleigh-Bénard convection in a laterally infinite cell was developed by Pellew and Southwell [1] and discussed by Chandrasekhar [2]. A revised version that applies to finite-cell convection was proposed by Sorokin $[3,4]$, and used extensively to investigate the influence of sidewalls on the stability by Chen [5]. However, in order to simplify the analysis, the dimensionless temperature $\theta$ of the fluid was assumed to satisfy the surrogate boundary condition (SBC) $\partial \theta / \partial n+\beta \theta=0$ on the sidewall, with $\partial / \partial n$ and $\beta$ representing the directional derivative along the outward normal $n$ and the effective thermal conductivity of the wall, respectively. Although this assumption has been quite common in this field, and it does capture the basic physics involved and can be realized in some simplified cases, it still is desirable to incorporate the sidewalls directly into the analysis to make the predictions completely general. It is the purpose of this paper to show that the inclusion of the sidewalls still leads to a variational formalism that is equivalent to solving a selfadjoint eigenvalue problem. Several results concerning the effects of the sidewalls are then derived via the com- bination of this formalism and perturbation theory. Finally I turn to the question of comparing the true system with a surrogate one and show that in a mathematically well-defined sense the real system's stability always lies in between that of two surrogate systems, thus justifying the use of the SBC in simulating the sidewall.

Let $\kappa, v$, and $\gamma$, respectively, stand for the thermal diffusivity, kinematic viscosity, and coefficient of thermal expansion of a layer $\Omega$ of pure fluid uniformly heated from below by a temperature difference $\Delta T$ across two horizontal plates separated by a distance $d$. The sidewall is denoted by $\Omega_{w}$. Define the Rayleigh number $R=\gamma g d^{3} \Delta T /(\kappa v)$, the Prandtl number $\sigma=v / \kappa$, and scale the length, time, and temperature, respectively, by $d, d^{2} / \kappa$, and $\kappa v \gamma g d^{3} \sqrt{R / \sigma}$; then the linearized NavierStokes equation under the Oberbeck-Boussinesq approximation and the linearized heat-transfer equation describing the deviation from the purely conducting state read

$$
\begin{aligned}
\frac{\partial \Psi}{\partial t}= & \mathcal{L} \Psi \\
\equiv & {\left[\left(\begin{array}{lllll}
\sigma & 0 & 0 & 0 & 0 \\
0 & \sigma & 0 & 0 & 0 \\
0 & 0 & \sigma & 0 & 0 \\
0 & 0 & 0 & 1 & 0 \\
0 & 0 & 0 & 0 & \kappa_{w}
\end{array}\right] \nabla^{2}\right.} \\
& \left.+\left(\begin{array}{ccccc}
0 & 0 & 0 & 0 & 0 \\
0 & 0 & 0 & 0 & 0 \\
0 & 0 & 0 & \sqrt{\sigma R} & 0 \\
0 & 0 & \sqrt{\sigma R} & 0 & 0 \\
0 & 0 & 0 & 0 & 0
\end{array}\right]\right) \Psi-\left(\begin{array}{c}
\nabla p \\
0 \\
0
\end{array}\right] \\
\equiv & \left(\hat{D}^{\prime} \nabla^{2}+\sqrt{\sigma R} \hat{A}\right) \Psi-\left(\begin{array}{c}
\nabla p \\
0 \\
0
\end{array}\right) \\
\boldsymbol{\nabla} \cdot \mathbf{u}=0 & \text { in } \Omega,
\end{aligned}
$$

where $\hat{D}^{\prime}$ and $\hat{A}$ are $5 \times 5$ matrices defined in an obvious way, and 


$$
\Psi \equiv\left(\begin{array}{c}
\mathbf{u} \\
\theta \\
\theta_{w}
\end{array}\right) \equiv\left(\begin{array}{c}
\psi \\
\theta_{w}
\end{array}\right)
$$

Here $\mathbf{u}, p$, and $\theta$ are the dimensionless velocity, pressure, and temperature of the fluid, respectively, while $\theta_{w}$ is the sidewall temperature. Also, $\kappa_{w}$ stands for the dimensionless thermal diffusivity of the sidewall. If we denote the ratio of the heat capacity per unit volume of the sidewall and the fluid by $r$, then across the interface $S$ of $\Omega$ and $\Omega_{w}$ the boundary conditions for $\Psi$ are

$$
\begin{aligned}
& u_{n} \equiv \mathbf{u} \cdot \hat{\mathbf{n}}=0, \\
& \frac{\partial \mathbf{u}_{\|}}{\partial n}+\alpha \mathbf{u}_{\|}=0, \\
& \theta=\theta_{w}, \\
& \frac{\partial \theta}{\partial n}=r \kappa_{w} \frac{\partial \theta_{w}}{\partial n},
\end{aligned}
$$

where $\mathbf{u}_{\|}$and $\partial \mathbf{u}_{\|} / \partial n$ are the shorthand notations for the projection of $\mathbf{u}$ and $\partial \mathbf{u} / \partial n$ onto the boundary, respectively, and $\alpha$ is a conveniently introduced non-negative constant that can differ on either the horizontal plate or the sidewall. (The physically admissible no-slip condition corresponds to taking $\alpha=\infty$ ). I will assume the outer surface $S_{2}$ of the sidewall is insulated and $\theta=\theta_{w}=0$ on both top and bottom plates. The two horizontal plates are assumed rigid (see Fig. 1).

After integration by parts it is easy to show that the operator $\mathcal{L}$ is self-adjoint, provided we define the inner product between $\Psi^{\prime}$ and $\Psi$ by

$$
\left\langle\Psi^{\prime} \mid \Psi\right\rangle \equiv \int_{\Omega} \psi^{\prime \dagger} \cdot \psi+\int_{\Omega_{w}} r \theta_{w}^{* *} \cdot \theta_{w},
$$

where the dagger indicates the complex conjugate of the transposition of a vector. Thus, the eigenvalues of $\mathcal{L}$ are real; and to determine the onset Rayleigh number one can simply set $\partial \Psi / \partial t=0$. Associated with $\mathcal{L}$ is a variational principle whose restricted version is discussed in detail in Ref. [5]. In this paper I will work directly with the properties of the critical Rayleigh number $R_{c}$ instead.

Absorbing $\sqrt{\sigma}$ into $\mathbf{u}$, one sees from Eq. (1) that $\sqrt{R_{c}}$ is a generalized eigenvalue of

$$
R \Psi \equiv-\nabla^{2} \Psi+\left(\begin{array}{c}
\nabla p \\
0 \\
0
\end{array}\right)=\lambda \hat{A} \Psi
$$

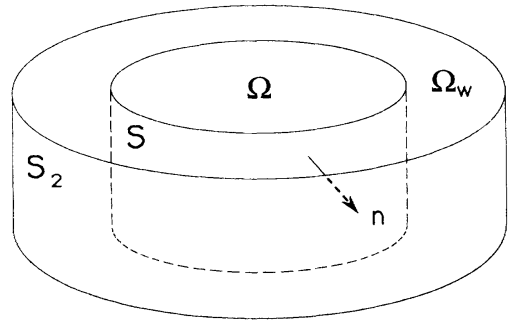

FIG. 1. Geometry of a convection cell and its sidewall.
Let $\hat{D}$ be a $5 \times 5$ diagonal matrix whose diagonal elements are $1,1,1,1$ and $r \kappa_{w}$, respectively. Then $R$ is an invertible operator because $\hat{D} \mathscr{R}$ is positive definite in the inner product defined above. If we now define a second inner product

$$
\left\langle\Psi^{\prime} \mid \Psi\right\rangle_{2} \equiv\left\langle\nabla \Psi^{\prime}|\hat{D}| \nabla \Psi\right\rangle+\int_{\partial \Omega} \alpha \mathbf{u}^{\prime *} \cdot \mathbf{u},
$$

then after integration by parts it is straightforward to show that the operator $R^{-1} \hat{A}$ is self-adjoint under the second inner product, and the eigenfunctions can be chosen to be real, as will be done from now on. In fact, for a given eigenstate $\Psi$ one finds

$$
\begin{aligned}
\frac{1}{\lambda} & =\frac{\left\langle\Psi\left|\mathcal{R}^{-1} \hat{A}\right| \Psi\right\rangle_{2}}{\langle\Psi \mid \Psi\rangle_{2}} \\
& =\frac{2 \int_{\Omega} u_{z} \theta}{\int_{\Omega}|\nabla \mathbf{u}|^{2}+|\nabla \theta|^{2}+\int_{\partial \Omega} \alpha|\mathbf{u}|^{2}+\int_{\Omega_{w}} r \kappa_{w}\left|\nabla \theta_{w}\right|^{2}} \\
& \equiv J[\Psi],
\end{aligned}
$$

a result that is trivially true in view of Eq. (8). Therefore, solving for $R_{c}$ is equivalent to solving a self-adjoint eigenvalue problem $(1 / \lambda) \Psi=\mathcal{R}^{-1} \hat{A} \Psi$, and the existence and completeness of the eigenstates (under the second inner product) follow standard arguments in the literature [6]. It is apparent that the problem is also equivalent to the following variational principle: for any trial function $\Psi$ that satisfies the incompressibility condition Eq. (2), the boundary conditions (BC's) Eqs. (4) and (6), and $\theta=\theta_{w}=0$ on the top and bottom plates, the functional $J[\Psi]$ takes a stationary value if and only if $\Psi$ is an eigenfunction of Eq. (8) that satisfies all the prescribed BC's and $R_{c}$ is associated with the largest positive eigenvalue. I will call the eigenstate associated with $R_{c}$ the "ground state." This variational characterization of $R_{c}$ enables one to deduce the monotonic dependence of it on the parameters in the problem. For instance, if we denote the ground-state wave function corresponding to two slightly different $\alpha$ and $\alpha^{\prime}$ by $\Psi$ and $\Psi^{\prime}$, respectively, then

$$
\begin{aligned}
R_{c}^{\prime-1 / 2}-R_{c}^{-1 / 2} & =J^{\prime}\left[\Psi^{\prime}\right]-J[\Psi] \\
& =\left(J^{\prime}\left[\Psi^{\prime}\right]-J^{\prime}[\Psi]\right)+\left(J^{\prime}[\Psi]-J[\Psi]\right) \\
& =O\left(\left(\alpha^{\prime}-\alpha\right)^{2}\right)+\frac{\partial J}{\partial \alpha}\left(\alpha^{\prime}-\alpha\right),
\end{aligned}
$$

where the first term is of second order in $\alpha-\alpha^{\prime}$ by the variational principle, and the states were assumed nondegenerate for simplicity. Because $\partial J / \partial \alpha$ is nonpositive when $J$ is maximized, we see the system is more stable, i.e., $R_{c}$ increases, if we make the boundary more rigid. Similarly, larger thermal diffusivity $\kappa_{w}$ renders higher stability. Note that for a thin wall with thickness $\tau$ much less than 1 and the radius of curvature of the interface $S$ we can approximate the temperature inside the wall by a quadratic in the (outward) radial coordinate $\xi$ that is zero on $S$ by definition:

$$
\theta_{w}=\frac{-\xi^{2}+2 \xi \tau}{2} \nabla_{S}^{2} \theta+\theta
$$


where $\theta$ is the fluid temperature at $\xi=0$ and $\nabla_{S}^{2}$ is the two-dimensional Laplacian on $S$. Equation (6) then becomes

$$
\frac{\partial \theta}{\partial n}=r \kappa_{w} \tau \nabla_{S}^{2} \theta .
$$

When $\nabla_{S}^{2}$ can be replaced by a negative number, as is the case if one considers a particular Fourier mode, then the $\mathrm{SBC} \partial \theta / \partial n+\beta \theta=0$ is recovered. This suggests the surrogate system must be closely related to the real system. The variational principle for a system subject to the SBC is still given by Eq. (9), with $\int_{\Omega_{w}} \kappa_{w}\left|\nabla \theta_{w}\right|^{2}$ replaced by $\int_{S} \beta \theta^{2}$. I will denote the variational functional associated with the SBC by $J^{\mathrm{SBC}}[\psi]$.

Notice that thickening the wall (not necessarily uniformly) by moving the outer boundary $S_{2}$ outward while keeping everything else fixed also increases the stability. This is because we can construct a trial function $\Psi$ for the smaller system from the ground state $\Psi^{\prime}$ of the larger system by defining $\Psi \equiv \Psi^{\prime}$ in their common domain of definition, and

$$
\sqrt{R_{c}^{\prime}}=1 / J\left[\Psi^{\prime}\right]=(1 / J[\Psi])+\delta_{p} \geq \sqrt{R_{c}} .
$$

Here, $\delta_{p}$ is a non-negative number that is proportional to the integral of $\kappa_{w}\left|\nabla \theta_{w}\right|^{2}$ in the newly created wall region. On the other hand, the stability is independent of the wall thickness when $\kappa_{w}$ is 0 or $\infty$ because the sidewall temperature is completely driven by the self-governed convection cell alone. In either case we can analyze the effects of the shape or size of the cell on the stability without reference to the sidewall. It can be shown that for the no-slip perfectly conducting case $R_{c}$ is strictly decreasing when one enlarges the cell [5].

Because $\theta_{w}$ is coupled to the convection cell solely through the cell-wall interface $S$, it is also possible to eliminate it in favor of fluid variables alone when one is considering the onset configuration. Let $g_{1}\left(\mathbf{r}, \mathbf{r}^{\prime}\right)$ be the Green's function for $-\nabla^{2}$ inside the wall with vanishing $\partial g_{1} / \partial n^{\prime}$ on $S_{2}$ and $g_{1}$ on the remainder of $\partial \Omega_{w}$. Then $\theta_{w}=\int_{S}\left(\partial g_{1} / \partial n^{\prime}\right) \theta^{\prime}$, where $\mathbf{n}$ is the outward normal to the cell and primed quantities are dummy variables in differentiation or integration. This allows us to rewrite the variational principle [Eq. (9)] in terms of fluid variables alone when $\theta_{w}$ is substituted by the expression derived above. We can also use another Green's function $g_{2}$ which satisfies the same BC's as $g_{1}$ except $\partial g_{2} / \partial n^{\prime}=0$ on $S$ to yield $\theta_{w}=-\kappa_{w}^{-1} \int_{S} g_{2}\left(\partial \theta^{\prime} / \partial n^{\prime}\right)$. The variational principle that explicitly incorporates $g_{1}$ is convenient for the case when $\kappa_{w}$ is small while for large $\kappa_{w}$ it is more appropriate to use $g_{2}$.

In the following I will first describe how one can use perturbation theory to derive the large-cell scaling property for the critical Rayleigh number $R_{c}^{\mathrm{SBC}}$ when the convection cell is subject to the SBC, and then I will show that this knowledge together with a simple application of the variational principle derived above immediately implies that the critical Rayleigh number for a cell surrounded by a real sidewall necessarily obeys the same scaling law. The argument hinges upon a key inequality
[Eq. (16)], which itself suggests the possibility of establishing a similar inequality [Eq. (20)] that is later shown to be true by virtue of the variational principle. These two inequalities then provide us with a transparent way of viewing the true $\mathrm{BC}$ as something that is midway between two SBC's, thus verifying our intuition that the SBC does capture the basic physics intrinsic to the case when a real sidewall is present.

At first sight, it seems that one must find out the explicit analytic expression of $R_{c}^{\mathrm{SBC}}$ for a cell of arbitrary geometry from Eq. (8) before one can extract its large-cell scaling property. But it is also obvious that this task is quite hopeless in general. To compromise, one might wish to try the standard trick of the method of separation of variables, hoping that under some suitable (yet possibly artificial) boundary conditions the method would work, thus allowing one to achieve the original goal. As it turns out, this indeed is the case for the problem at hand. This being done, the case with the physically more relevant BC's can then be handled by perturbation theory, and useful information about this system extracted. This is the approach I will adopt in the following derivation.

In order to solve the fluid equations by the method of separation of variables, it turns out that the sidewall should be perfectly insulating and the vertical vorticity $\omega$ must vanish $[5,7]$. For the marginally stable state we have $\nabla^{2} \omega=0$. After multiplying this equation by $\omega$ and integrating over $\Omega$ one finds $\omega$ is identically zero when the fluid is irrotational at the sides and the two horizontal plates satisfy Eqs. (4) and (5). (This "rotation-free" condition is different from Eq. (5) by an extra term, which involves the curvature of the sidewall. The technical detail of this point is discussed in Ref. [5].) Therefore, Eq. (8) can be solved by separation of variables if the sidewall is a "rotation-free" perfect insulator, and the eigenfunction is of the form

$$
\begin{aligned}
& u_{x}=\frac{d \bar{u}}{d z} \frac{\partial \phi}{\partial x}, \quad u_{y}=\frac{d \bar{u}}{d z} \frac{\partial \phi}{\partial y}, \quad u_{z}=\mu \bar{u} \phi \\
& \theta=\bar{\theta} \phi, \quad p=\left(\frac{d^{3} \bar{u}}{d z^{3}}-\mu \frac{d \bar{u}}{d z}\right) \phi
\end{aligned}
$$

where $\bar{u}$ and $\bar{\theta}$ are functions of $z$ to be determined by the fluid equations and the BC's at $z=0,1$, and $\phi$ is any nonconstant Neumann eigenfunction of the two-dimensional Laplacian on the horizontal cross section of the cell: $\nabla^{2} \phi=-\mu \phi$. Treating $R_{c}$ as a function of $\mu$, one finds that its minimum $R_{c \infty}$ corresponds to some finite $\mu_{c}$. Because $\mu$ scales in size $L$ like $L^{-2}$, we see that different $\mu$ 's will pass through $\mu_{c}$ sequentially to render $R_{c}$ minimum as we increase $L$. The $R_{c}$ vs $L$ curve $\Gamma_{c}$, therefore, looks like the bottom curve in Fig. 2. The positions of the cusps in this figure can be determined analytically, and they fall on a monotonically decreasing curve $\Gamma$ which behaves like $R_{c}-R_{c \infty} \propto L^{-2}$ for large $L$. If we tune the $\alpha$ and/or $\kappa_{w}$ of the sidewall to any positive value or even infinity, the cusps will only be perturbed by an amount of order $L^{-3}$. However, the oscillations of $\Gamma_{c}$ are immedi- 


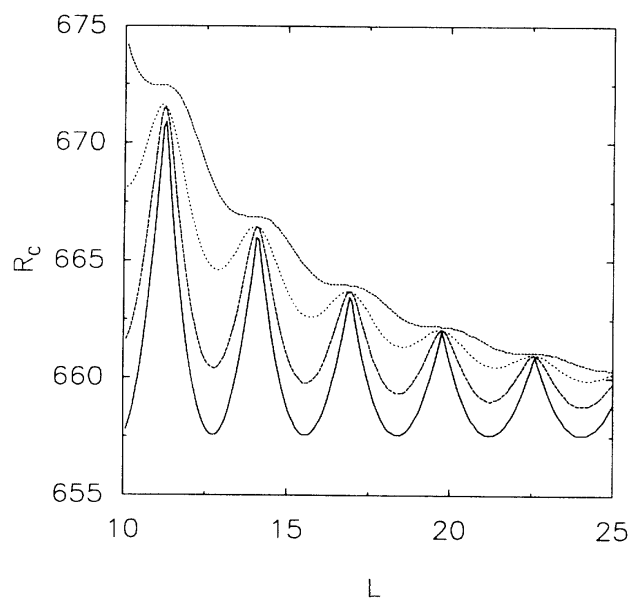

FIG. 2. Critical Rayleigh number $R_{c}$ vs (dimensionless) cell size $L$ for a two-dimensional free-slip box whose sidewall thickness $\tau \ll<1$. Sidewall parameters are (from bottom curve to top) $\alpha=r \kappa_{w} \tau \pi^{2}=0,0.2,1$, and 100 , respectively.

ately destroyed upon perturbation for all large enough $L$ so that the full curve will not only never dip to $R_{c \infty}$ again but will almost coincide with $\Gamma$ for all $L$ greater than some crossover length $L_{\mathrm{CO}}$. This crossover size depends inversely on the strength of the perturbation: the weaker the perturbation, the larger $L_{\mathrm{CO}}$ becomes (Fig. 2). For a system subject to the SBC, this feature can be derived on the following basis [5]. For a cylindrical cell we can consider eigenstates of the same azimuthal quantum number $m$. From perturbation theory one discovers that near the sidewall one of the two perturbed eigenstates at the cusp is $O\left(L^{-1}\right)$ smaller than its bulk value. This in turn implies its corresponding eigenvalue is perturbed by an amount $O\left(L^{-1}\right)$ smaller than that for systems whose sizes are not "right." (The size is "right" if a cusp is present.) Because the total perturbation in $R_{c}$ for systems of any (large enough) size can only be of $O\left(L^{-2}\right)$ [5], we see these "anchored" states only change by $O\left(L^{-3}\right)$ under perturbation. Now consider a cell with a rigid, perfectly conducting sidewall. Its monotonic dependence of $R_{c}$ on $L$ and the previous conclusion implies that the $R_{c}$ vs $L$ curve $\Gamma_{c}$ for this case must coincide with $\Gamma$ [correct to $O\left(L^{-2}\right)$ ]. Perturbing from this most stable state apparently will not change this characteristic for all large enough $L$, and the claims are verified. (This prediction is actually quite clear from the numerical work done by Charlson and Sani [8], though they did not explicitly discuss the origin of this feature. I have also verified that their data do confirm the prediction of the $L^{-2}$ scaling law [5].) For a large cell with other geometry, we can smoothly deform a cylindrical cell to the desired shape and show that the claims still hold, provided the radius of curvature of the sides is no smaller than $O(L)$. For a cell surrounded by a real sidewall, instead of being subject to the SBC, one can arrive at the same conclusions by similar reasoning. But there is another way of looking at this matter which allows us to see the connection between the SBC and the real BC more closely.
Let $\Psi$ be the ground state for a cell with a real sidewall; then

$$
\begin{aligned}
\frac{\int_{\Omega_{w}}\left|\nabla \theta_{w}\right|^{2}}{\int_{S} \theta^{2}} & \left.=\left[\frac{\int_{\Omega_{w}}\left|\nabla \theta_{w}\right|^{2}}{\int_{\Omega_{w}} \theta_{w}^{2}}\right] \mid \frac{\int_{S} \theta_{w}^{2}}{\int_{\Omega_{w}} \theta_{w}^{2}}\right]^{-1} \\
& \geq \frac{\zeta}{c_{1} \sqrt{\xi}+c_{2}} \geq c
\end{aligned}
$$

for some constants $c_{1}, c_{2}$, and $c$, where $\zeta$ stands for the expression inside the first pair of parentheses, which is $\geq \pi^{2}$, and the BC $\theta=\theta_{w}$ and an inequality bounding the second pair of parentheses by $\sqrt{\xi}$ are exploited [5]. If we define $\beta \equiv c r \kappa_{w}$ for a given $\kappa_{w}$ and denote the restriction of $\Psi$ on $\Omega$ by $\psi$, then

$$
R_{c}^{\mathrm{SBC}^{-1 / 2}} \geq J^{\mathrm{SBC}}[\Psi] \geq J[\Psi]=R_{c}^{-1 / 2},
$$

i.e., the critical Rayleigh number for the real system is bounded below by that for a surrogate system with $\beta=c r \kappa_{w}$, irrespective of whatever non-negative value $\kappa_{w}$ takes. This immediately implies $R_{c}-R_{c \infty}=O\left(L^{-2}\right)$ if the thickness of the sidewall remains about the same when one increases $L$. In fact, we can also bound $R_{c}$ from above by another surrogate system with $\beta=c^{\prime} r \kappa_{w}$ for some constant $c^{\prime}$. I shall show that actually this is true for a cell with an infinitely extended sidewall. Then the statement is certainly true for a cell with a finite sidewall because I showed its $R_{c}$ is even smaller. Let $\psi$ be the ground state of the surrogate cell. Define a coordinate system $\xi-\eta$ in the wall region such that $\xi$ is the arc length of the "radial coordinate" that is orthogonal to $S$ and vanishes on $S$, whereas $\eta$ is the coordinate on the two-dimensional surface $S^{\prime}$ defined by $\xi=$ const. I will assume the coordinates are chosen so that the angle between the $\xi$ curve and the surface normal to $S^{\prime}$ is bounded away from $\pi / 2$. Construct a trial function $\Psi$ for the real system with a laterally infinite sidewall by extending the definition of $\psi$ into the wall region so that

$$
\begin{aligned}
\theta_{w} & \equiv\left[\theta+\frac{1}{r \kappa_{w}} \frac{\partial \theta}{\partial n} f_{1}\right] f_{2} \\
& =\theta\left(1-\frac{\beta}{r \kappa_{w}} f_{1}\right] f_{2} \\
& \equiv \theta\left(1-\zeta f_{1}\right) f_{2} \\
& \equiv \theta h f_{2},
\end{aligned}
$$

where $f_{2}$ is a function of $\xi$ that satisfies $f_{2}^{\prime}(0)=0$, $f_{2}(0)=1$, and decays sufficiently fast to zero as $\xi$ tends to $\infty$, while $f_{1} \equiv \xi$ except when $\xi$ is greater than some prescribed number $\delta$, in which case we define $f_{1} \equiv \delta$. Also, $\theta$ is the fluid temperature on $S$ whose definition can be trivially extended into the whole wall region by $\left.\theta(\xi, \eta) \equiv \theta(\eta)\right|_{S}$. Then 


$$
\begin{aligned}
\int_{\Omega_{w}}\left|\nabla \theta_{w}\right|^{2}= & \int_{\Omega_{w}}\left|h f_{2} \nabla \theta+\theta f_{2} \nabla h+\theta h \nabla f_{2}\right|^{2} \\
\leq & h_{M}^{2} \int_{\Omega_{w}}|\nabla \theta|^{2} f_{2}^{2}+\xi^{2} \int_{\xi<\delta} \theta^{2} f_{2}^{2}+h_{M}^{2} \int_{\Omega_{w}} \theta^{2}\left|\nabla f_{2}\right|^{2} \\
& +2 \xi h_{M} \int_{\xi<\delta}|\theta \nabla \theta| f_{2}^{2}+2 h_{M}^{2} \int_{\Omega_{w}}|\theta \nabla \theta|\left|f_{2} \nabla f_{2}\right|+2 \xi h_{M} \int_{\xi<\delta} \theta^{2}\left|f_{2} \nabla f_{2}\right| \\
\leq & a\left[h_{M}^{2} \int_{S}\left|\nabla_{S} \theta\right|^{2}+\left(h_{M}+\delta_{1} \zeta\right)^{2} \int_{S} \theta^{2}+2\left(\delta_{1} \zeta+h_{M}\right) h_{M}\left[\int_{S} \theta^{2} \int_{S}\left|\nabla_{S} \theta\right|^{2}\right]^{1 / 2}\right],
\end{aligned}
$$

where $h_{M}$ is the maximum of $h$, and $a$ is some constant depending on the geometry of the cell and the coordinates we choose. In the above, $\delta_{1}$ is a number that is proportional to $\sqrt{\delta}$ when $\delta$ is very small. Also, $\nabla_{S}$ is the gradient operator on the interface $S$. But we observe that for the ground state it is possible to find a constant $b$ such that

$$
\int_{S}\left|\nabla_{S} \theta\right|^{2} \leq b^{2} \int_{S} \theta^{2}
$$

for all $\beta$. First of all, this statement is trivially true if it so happens that $\theta$ is identically zero on $S$ for all $\beta$. So we may consider the case when $\theta$ is identically zero on $S$ only at some isolated points $\beta_{0}$ of $\beta \in[0, \infty)$. (The case $\beta_{0}=\infty$ is included in this argument.) Near each of these points we can expand $\theta$ in a power series of a small pa- rameter $\delta^{\prime}$. (We can take $\delta^{\prime} \equiv \beta-\beta_{0}$ if $\beta_{0}$ is finite; the case $\beta_{0}=\infty$ can be handled by taking $\delta^{\prime} \equiv \beta^{-1}$.) Suppose $\theta=\theta_{n} \delta^{\prime n}+\cdots$ for some leading power $n$ such that $\theta_{n}$ is not identically zero on $S$; then clearly Eq. (18) is satisfied for some $b$. Also, for $\beta$ outside the neighborhood centered at $\beta_{0}$ we know the left-hand side of Eq. (18) must be bounded above because the eigenfunction is twice differentiable, while the right-hand side is bounded away from zero by construction. And so a constant $b$ certainly can be found to satisfy Eq. (18). Therefore Eq. (17) can be further reduced to

$$
\int_{\Omega_{w}}\left|\nabla \theta_{w}\right|^{2} \leq a\left(b h_{M}+h_{M}+\delta_{1} \zeta\right)^{2} \int_{S} \theta^{2} .
$$

But one easily verifies that

$$
c^{\prime} \equiv \frac{a^{-1}-2(b+1) \delta_{1}-\sqrt{\left[a^{-1}-2(b+1) \delta_{1}\right]^{2}-4(b+1)^{2} \delta_{1}^{2}}}{2 \delta_{1}^{2}}
$$

is a positive solution to the following equation for $\zeta$ if $\delta$ is small enough:

$$
a\left(b h_{M}+h_{M}+\delta_{1} \zeta\right)^{2}=a\left(b+1+\delta_{1} \zeta\right)^{2}=\zeta .
$$

Choose the $\beta$ for the surrogate system to be $c^{\prime} r \kappa_{w}$; then the previous reasoning immediately implies

$$
r \kappa_{w} \int_{\Omega_{w}}\left|\nabla \theta_{w}\right|^{2} \leq \beta \int_{S} \theta^{2},
$$

which in turn tells us

$$
R_{c}^{\mathrm{SBC}^{-1 / 2}} \equiv J^{\mathrm{SBC}}[\Psi] \leq R_{c}^{-1 / 2}
$$

by virtue of the variational principles for both systems. Again, I must emphasize that this inequality holds for all non-negative $\kappa_{w}$.
In conclusion, the linear stability of pure-fluid convection with realistic boundary conditions can be analyzed by the combination of perturbation theory and variational formalism. The theory not only predicts the scaling behavior for $R_{c}$ but also claims that replacing the sidewall thermal property by the mathematically simpler SBC does capture the underlying physics because, as far as the onset instability is concerned, in a well-defined sense the real system is sandwiched between two surrogate systems.

Constant inspiration and encouragement from Professor M. C. Cross have made this work all the more enjoyable. I also thank Professor N.-C. Yeh and Professor H. B. Keller for useful discussions. This work is supported by NSF Grant No. DMR-9013984.
*Present address: Institute of Physics, Academia Sinica, Nankang, Taipei, Taiwan, Republic of China.

[1] A. Pellew and R. V. Southwell, Proc. R. Soc. (London) Ser. A 176, 312 (1940).

[2] S. Chandrasekhar, Hydrodynamic and Hydromagnetic Stability (Oxford University Press, London, 1961), p. 27ff.

[3] V. S. Sorokin, Prikl. Mat. Mekh. 17, 39 (1953).

[4] V. S. Sorokin and I. V. Sushkin, Zh. Eksp. Teor. Fiz. 38, 612 (1960) [Sov. Phys. JETP 11, 440 (1960)].

[5] Y. -Y. Chen, J. Fluid Mech. (to be published).

[6] For an elementary treatment of the simpler Laplacian operator that actually can be generalized to our problem, see P. R. Garabedian, Partial Differential Equations (Wiley, New York 1964), Chap. 10. For the treatments of the linearized Navier-Stokes equation, see O. A. Ladyzhenskaya, The Mathematical Theory of Viscous Incompressible Flow (Gordon and Breach, New York, 1963) and V. I. Yudovich, The Linearization Method in Hydrodynamical Stability Theory (American Mathematical Society, Providence, 1989).

[7] For more details and related matters, see Y. -Y. Chen, Ph.D. thesis, California Institute of Technology, 1991.

[8] G. S. Charlson and R. L. Sani, Int. J. Heat Mass Trans. 13, 1469 (1970). 